\title{
Sur une propriété des ensembles clairsemés.
}

$\operatorname{Par}$

\author{
W. Sierpińs ki (Varsovie).
}

M. Kuratowski a démontré récemment que toute infinité bien ordonnée d'ensembles clairsemés croissants (décroissants) est dénombrable 1). Dans cette Note nous démontrerons un théorème sur les familles quelconques d'ensembles clairsemés (d'un espace à $m$ dimensions), ordonné d'après leurs grandeurs, théorème dont on pourra déduire sans peine celui de M. Kuratowski.

Théorème: Une somme d'une infinité quelconquo d'ensembles clairsemés, tels que de tous deux un est contenu dans lautre, est effectivement énumérablo.

Démonstration. Soit $\mathcal{F}=\{C\}$ une famille d'ensembles clairsemés qui sont en relation d'inclusion (c'est à-dire, $C_{1}$ et $C_{2}$ étant deux ensembles appartenant aे $\mathfrak{F}$, un $\theta$ au moins de deux relations subsiste: $C_{1} C C_{2}$ et $\left.C_{2} \subset C_{1}\right)$. Désignons par $S=\Sigma C$ la somme de tous les ensembles $C$ constituant $\mathscr{F}$. Tout ensemble clairsemé est, comme j'ai démontré ailleurs effectivement énumérable ${ }^{2}$ ). Rour démontrer notre théorème il suffira donc supposer que l'ensemble $S$ n'est pas clairsemé: dans ce cas il contient un sous-ensemble dense en soi $D$ qui y est le plus grand.

Soit $C$ un ensemble donné appartenant a $\mathscr{F}, P=\Pi Q \quad \ldots . .$. un produit dont les facteurs appartiennent al $d$. Je dis que $C$ et $P$ sont en relation d'inclusion ${ }^{3}$ ). En effet, un des deux cas subsiste toujours: il existe un facteur $Q$ du produit $P$ contenu dans $C$, et

1) V. ce volume, p. 42, note 1 ).

2) V. Fund. Math. t. 1, p. 2.

ง) Nous conviendrons ici de regarder un ensemble vide comme contenu dans tout ensemble. 
ulors on a $P \subset C$, ou bien un tel facteur n'existe pas, et alors (tout $Q$ et $C$, comme ensembles appartenant à $\mathscr{F}$, étant dans la rolation d'inclusion) on a $Q \supset C$ pour tout facteur $Q$ de $P$, d'où: $P \supset C$.

Nous pouvons, comme on sait, ranger dans une suite infinie bien déterminée toutes les sphères $m$-dimensionnelles dont les rayons sont rationnels et dont les centres ont des coordonnées rationnelles. Soient $S_{n}(n=1,2,3, \ldots)$ les sphères consécutives de cette suite qui ont des points communs avec l'ensemble $D$. Nous aurons donc $S_{n} D \neq 0$ pour $n=1,2,3, \ldots$ par conséquent ( $D$ étant contenu dans $S=\Sigma C$ ) il existe, pour tout indice $n$ donné, un ensemble $C$ do la famille ef tel que $S_{n} D C \neq 0$ : nous désignerons (pour $n$ donné) par $P_{n}$ lo produit de tous ces ensembles $C$. Je dis que $S=P_{1}+P_{2}+P_{3}+\ldots$

En offet, posons $T=P_{1}+P_{2}+\ldots$ et admettons que $S \neq T$. Nous avons toutefois $\left.S_{\ldots}\right) P_{n}(n=1,2 \ldots)$, done $S \supset T$. Par conHéquent, si $S \neq T$, il existe (d'après $S=\Sigma C$ ) un ensemble $C_{0}$ de la famille a qui n'est pas contenu daris $T$ : un tel ensemble $C_{0}$ no vérifie non plus la relation $C_{0} \subset P_{n}$ pour aucun $n$ naturel. Or, comme nous savons, $C_{0}$ et $P_{n}$ sont en relation d'inclusion: il est done $C_{0} \supset P_{u}$ pour $n=1,2,3, \ldots$, d'où $C_{0} \supset P_{1}+P_{z}+\ldots=T$.

Jo dis que l'ensemble TT est dense dans $D$. In effet, soit $p$ un point de $D, H \ldots$ l'intérieur d'une sphère quelconque entourunt $p$. L'onsomble $H D$ (comme coutenant $p$ ) n'est pas vide: donc (d'uprès $D C S$ ), nous avons $H D S \neq 0$ et il existe un ensemble $C_{1}$ de la famille dr tel que $H D C_{1}=0$. Or, $H D C_{1}$, comme sousonsemble de l'ensemble clairsemé $C_{1}$, est clairsemé, donc contient un point isolé, $q$. Or, $q$ étant un point isolé de $C_{1}$ intérieur à $H$, il existe une sphère rationnelle intérieure à $H$, contenant à son interrieur $q$ et ne contenant aucun point de l'ensemble $H D C_{1}$, autre que q. Co sera évidemmenti une sphère de la suite $S_{n}$, soit $S_{\mathrm{k}}$ (car c'est uno sphère rationuelle uyant un point cornmun $q$ avec $D$ ). Il est elair que l'onsemble $P_{\text {tit }}$ no compose d'un seul point, $q$ (puisque l'ensemble $S_{k} D C_{1}=S_{h} D C_{1} H$ se réduit au point $q$ et puisque il en est de même pour les onsombles $O C^{\prime} C_{1}$ pour lasquels $\left.S_{k} D C \neq 0\right)$. Donc $T=\sum_{n} P_{n}$ contient lo point $q$ (qui appartient a $F D$ ). Nous avons done prouvé que tont entourage du point $p$ de $D$ contient un point de $T D$. L'ensemble $T$ T $D$ est donc dense dans D. L'ensemble $D$ étant dense 
en soi, il en résulte que $T D$ est dense en soi. L'ensemble clairsemé $C_{0} \supset T$ contiendrait done un sous-ensemble dense en soi $T D$, ce qui est impossible.

Nous avons donc $S=P_{1}+P_{2}+P_{8}+\ldots$ Or les ensembles $P_{n}(n=1,2, \ldots)$ comme produits d'ensembles clairsemés, sont clairsemés (ou vides). Il existe, comme on sait, une loi d'après laquelle on peut énumérer effectivement les éléments de tout ensemble clairsemé ${ }^{1}$ ): il en résulte que l'ensemble $S=P_{1}+P_{2}+\ldots$ est effectivement énumérable, c. q. f. d.

Notre théorème et ainsi démontré.

Il importe de remarquer que les ensembles $C$ constituant la famille $\mathscr{f}$ peuvent former une infinité nou dénombrable. Soit, en effet, $P$ un ensemble parfait linéaire non dense, $S$ - l'ensemble des centres de tous les intervalles contigus à l'ensemble $P$ : ce sera évidemment un ensemble isolé. Pour tout point $p$ de $P$ désignons par $C(P)$ l'ensemble de tous les points de $S$ situés à gauche de $p$ : les ensembles $C(p)$ seront donc tous isolés (donc clairsemés). On voit sans peine que lorsque $p$ et $q$ sont deux points de $P$ tels que $p<q$, on aura $C(p) \neq C(q)$ et $C(p) \subset C(q)$. La famille $\mathscr{F}$ constituée par tous les ensembles $C(p)$, où $p$ appartient à $P$, aura donc la puissance du continu. Donc:

Une infinité d'ensembles isolés, tels que de tous deax unest toujours contenu dans l'autre, peut être de puissance du continu.

Or on peut sans peine déduire de notre théorème la proposition que voici:

Uneinfinité bien ordonnée d'ensembles clairsemés croissants est effectivement énumérable. ${ }^{2}$ ).

Soit, en effet, $\mathscr{F}=\{C\}$ une telle infinité d'ensembles, $S=\Sigma C$ la somme des ensembles constituaut $\mathscr{F}$ : d'après notre théorème l'ensemble $S$ est effectivement énumérable. Nous savons done ranger les éléments de $S$ en une suite infinie

$$
s_{1}, s_{2}, s_{3}, \ldots
$$

Soit $C_{0}$ un ensemble donné appartenant à $\vec{F}$. $\mathrm{Si}^{\prime} C_{0}=S$, posons

1) V. Fund. Math. t. I, p. 5.

2) Une proposition analogap pour les ensembles clairsemés décroisaanta résulte immédiatement de la remarque que tout ensemble clairsemé est effectivement énumérable 
$\mu\left(C_{0}\right)=0$. Si $C_{0} \neq S$, il existe dans la somme $S=\Sigma C$ des termes plus grands que $C_{0}$ (e'est-à-dire des ensembles $C$ de $\mathcal{F}$ contenauts $C_{0}$ et non contenus dans $C_{0}$ ) et parmi eux un le plus petit $C^{*}$ (puizque les ensembles $C$ forment une famille $\mathscr{F}$ bien ordonnée d'après les grandeurs croissantes des $C$ ). Soit $s_{n}$ le premier terme de la suite (1) appartenant is $C^{*}-C_{0}$ : nous poserons $\mu\left(C_{0}\right)=n$. Ainsi à tout ensemble $C$ de $\mathscr{F}$ correspond un entier déterminé $\mu(C) \geqslant 0^{\circ}$ et il est clair qu'aux ensembles distincts correspondent des entiers différents (puisque, lorsque $C_{1} \subset C_{2}$ et $C_{1} \neq C_{2}, s_{\mu\left(C_{2}\right)}$ u'appartient a $C_{2}$ et $s_{\mu\left(c_{2}\right)}$ n'appartient pas à $C_{2}$ ). L'ensemble $\mathscr{F}$ est donc effectivement énumérable c. q. f. d.

Nous avons en même temps dérnontré le théorème de $\mathbb{M}$. $\mathbb{R}$ : ratowsi ans faire appel à l'axiome de M. Zermelo et n'utilisant pas les nombres transfinis. 